\title{
Ein Vorschlag des dialektbasierten Hoch- deutschunterrichts für die Wolgadeutschen in Argentinien
}

\author{
A proposal of dialect-based Standard German teaching for Volga Germans in Argentina \\ http://dx.doi.org/10.11606/1982-8837213431
}

\author{
Anna Ladilova ${ }^{1}$
}

\begin{abstract}
Just like many other German communities in the Americas the Volga Germans in Argentina have been able to maintain their initial dialectal variety of German until today. It has been passed on mostly orally, has only covert prestige and functions as an ethnic identity marker for the speakers. As opposed to that the language of the majority population (Spanish in the case of the Volga Germans in Argentina) has overt prestige and objective value for everyday life. Also the standard variety of German is perceived as objectively useful, e.g. for the future career. At the same time, the variety of German spoken by the community is perceived as incompatible with standard German: The speakers of the dialect often think that their knowledge of the dialect is more an obstacle for learning standard German than a help. Nevertheless, there have already been initiatives in Argentina of developing and implementing dialect-based standard German classes, e.g. by Arndt Schmidt in his program for training teachers in the Volga German village in Santa Maria in the province of Buenos Aires. The present article will discuss the question of German classes for dialect learners, give a short overview of the sociolinguistic situation of Volga Germans in Argentina and make a proposal of a heritage-dialect based standard German teaching program.
\end{abstract}

Keywords: Volga Germans in Argentina, heritage language preservation, German teaching, migration

Zusammenfassung: Wie in vielen anderen deutschen Gemeinschaften in Amerika wurde bei den Wolgadeutschen in Argentinien bis heute eine dialektale Varietät des Deutschen aufrechterhalten. Diese wurde i.d.R. nur mündlich weitergegeben, verfügt über covert prestige für die Sprecher und dient als Marker ethnischer Identität. Die Sprache der Mehrheitsgesellschaft (Spanisch im Falle der Wolgadeutschen in Argentinien) verfügt dagegen über overt prestige und objektiven Nutzen für den Alltag. Auch dem Hochdeutschen wird ein objektiver Nutzen, z.B. für die berufliche Zukunft, zugesprochen. Gleichzeitig wird die von der Gemeinschaft gesprochene Varietät meist als inkompatibel mit dem Hochdeutschen wahrgenommen: Die Sprecher des Dialekts sind oft der Meinung, dass die Dialektkenntnisse für sie eher ein Hindernis als eine Hilfe beim Erwerb des Hochdeutschen darstellen. Nichtsdestotrotz gab es bereits Initiativen dialektbasierten Hochdeutschunterricht für die Wolgadeutschen in Argentinien voranzutreiben: So initiierte z.B. Arndt Schmidt ein Programm zur Ausbildung der Lehrer im wolgadeutschen Dorf Santa Maria in der Provinz Buenos Aires. Der vorliegende Artikel geht auf die Frage des Deutschunterrichts für Dialektlerner ein, gibt einen kurzen Überblick über die soziolinguistische Situation der

\footnotetext{
${ }^{1}$ Justus-Liebig-Universität Giessen, Institut für Romanistik, Karl-Glöckner-Straße 21 G, 35394, Giessen, Germany. E-mail: aladilova@gmail.com
} 
Ladilova, A. - Wolgadeutsche in Argentinien

Wolgadeutschen in Argentinien und macht einen Vorschlag eines ursprungsdialektbasierten Hochdeutschunterrichts.

Stichwörter: Wolgadeutsche in Argentinien, Erhalt der Herkunftssprache, Deutschunterricht, Migration

\section{Einleitung}

Die Frage nach der Gestaltung des Deutschunterrichts für Dialektsprecher ${ }^{2}$ stellt sich nicht nur in deutschsprachigen Ländern, sondern genauso bei den deutschsprachigen Minderheiten in der Diaspora, die eine Dialektvarietät des Deutschen sprechen. So haben auch die in Argentinien lebenden Wolgadeutschen ihre ursprüngliche Mundart, das Wolgadeutsche, ${ }^{3}$ bis heute weitgehend aufrechterhalten, welche eine fruchtbare Basis für den Erwerb des Hochdeutschen darstellt. Beim Deutschunterricht wurde diese bisher von den Lehrern aber als störend für den Spracherwerb abgelehnt. Dies, sowie die Tatsache, dass die Wolgadeutschen in Argentinien großes Interesse daran haben das Deutsche ${ }^{4} \mathrm{zu}$ lernen (vgl. FEICK 2005; LADILOVA 2013), macht die Aktualität und die Notwendigkeit eines auf die sprachliche Ausgangssituation der Wolgadeutschen angepassten Unterrichtskonzepts deutlich.

Der vorgestellte Unterrichtsentwurf ${ }^{5}$ ist an die auf dem Land lebenden Wolgadeutschen in Argentinien gerichtet, die in Dörfern mit ca. 1000-1500 Einwohnern leben. Diese verfügen i.d. R. nicht über die notwendigen finanziellen Mittel, um Deutschunterricht für verschiedene Altersgruppen anzubieten, und die Implementierung von Deutschunterricht in den schulischen Lehrplänen ist in den meisten Dörfern ebenfalls noch nicht möglich. Es wird daher sowohl in Bezug auf das Alter (zwischen 14 und 45 Jahren), als auch in Bezug auf die Kenntnisse des Wolgadeutschen (die Älteren sprechen es i. d. R. besser als die Jüngeren) von heterogenen Lernergruppen ausgegangen. Da die äußere Differenzierung nicht möglich ist, soll insofern eine innere Differenzierung

\footnotetext{
${ }^{2}$ Mit dem Begriff „Dialekt“ sind im Folgenden im Sinne von Ulrich AMMON (1983: 36) Sprachvarietäten gemeint, die kleinregional und nichtstandardisiert bzw. gesprochen sind.

${ }^{3}$ Diese Bezeichnung wird in der vorliegenden Arbeit für die von den Wolgadeutschen in Argentinien gesprochene Varietät des Deutschen verwendet. Diese stellt keineswegs eine homogene Varietät dar, sondern weist eine deutliche diatopische Variation in Argentinien auf.

${ }^{4}$ Insofern keine Präzisierung vorgenommen wird, sind im Folgenden mit der Bezeichnung "Deutsch" sowohl das Hochdeutsche wie auch die jeweilige/n Varietät/en des Deutschen gemeint.

${ }^{5}$ Dieser Entwurf wurde im Rahmen der Doktorarbeit der Verfasserin (Ladilova 2013) entwickelt und wurde noch nicht pilotiert.
} 
Ladilova, A. - Wolgadeutsche in Argentinien

stattfinden, als dass im Unterricht lernerorientiert gearbeitet wird. Der Unterricht soll einmal pro Woche stattfinden und eineinhalb Zeitstunden dauern. Darin soll das Wolgadeutsche nicht nur als Transferbasis zum Hochdeutscherwerb, sondern auch als eine Bereicherung für das sprachliche Repertoire der Lerner betrachtet werden. Zudem wird der Aufbau von Sprachbewusstheit - im Sinne von Reflektion über den eigenen Sprachgebrauch und des bewussten Wissens über Sprache und den Spracherwerb durch die Lerner - als eine gute Möglichkeit betrachtet, Sprachunterricht mit heterogenen Lerngruppen zu gestalten (BUDDE 2012: 7; ASSOCIATION FOR LANGUAGE AWARENESS s/d). ${ }^{6}$ Diese soll durch die Auseinandersetzung mit den Sprachstrukturen des Deutschen, den Abweichungen des Wolgadeutschen vom Hochdeutschen sowie mit dem spezifischen soziohistorischen Hintergrund der eigenen Gruppe gefördert werden.

Nach einer theoretischen Einbettung in das Thema Deutschunterricht für Dialektsprecher wird näher auf die Zielgruppe eingegangen, bevor das Unterrichtskonzept für die ersten 20 Stunden vorgestellt und ein Fokus auf eine Doppelstunde gelegt wird.

\section{Deutschunterricht für Dialektlerner}

Die Debatte um die Koexistenz von Dialekt und Standardsprache im Schulunterricht geht auf die im 18. und 19. Jahrhundert angestrebten Normierungen und Vereinheitlichungen von Nationalsprachen zurück, die die Forderung nach einer streng normierten Sprache für die Schule aufstellten. Doch schon Ende des 19. Jahrhunderts erhob sich der Widerspruch gegen die alleinige Dominanz der Standardsprache. Trotzdem setzte sich die Tendenz des Zurückdrängens der Dialekte im Deutschunterricht (vor allem in Norddeutschland) fort. In den sechziger und siebziger Jahren begann die sogenannte Sprachbarrierendiskussion, die unter Rückbezug auf die Defizithypothese von Bernstein den Dialektgebrauch als Grund für geringeren Erfolg der Schüler sah (vgl. NEULAND; HochHOLzER 2006: 176179).

Die Tatsache, dass dialektsprechende Kinder für Erwerb und Beherrschung der Standardsprache oft zusätzliche Leistungen bei der Unterscheidung von Dialekt und Standardsprache und ihren jeweiligen Regularitäten erbringen mussten, wurde zugleich aber auch als

\footnotetext{
${ }^{6}$ Zur tiefergehende Auseinandersetzung mit dem Begriff der Sprachbewusstheit siehe BUDDE (2012), KÄFER (2013), LUCHTENBERG (2010), NEULAND (2002). 
Ladilova, A. - Wolgadeutsche in Argentinien

ein Beleg gegen eine sprachliche oder gar geistige Minderausstattung dialektsprechender Kinder ausgelegt (NEULAND; HOCHHOLZER 2006: 179).

So können bei DialektsprecherInnen tatsächlich verstärkt Fehler in der Orthographie, Grammatik und Lexik sowie Schwierigkeiten bei der Textproduktion und -organisation und beim Wechsel zwischen formalem und informellem Sprachgebrauch auftreten (vgl. STRUGER 2008: 46). Als Gegenmittel wurde ein kompensatorischer Sprachunterricht empfohlen, der den Dialekt als „defizitäres Sprachsystem angesehen, dementsprechend stigmatisiert und als minderwertig“" (SCHIEßL 2009: 34) abgelehnt hatte.

Auch die Auslandsgermanistik ist durch die Ausrichtung nach der standardsprachlichen Norm geprägt, was nicht zuletzt damit zu tun hat, dass „,im Lernprozess das Lernen nach der Schrift erfolgt und die standardsprachliche Norm durch prä- und deskriptiven Regeln eine einheitliche, Abweichungen nur in geringem Maße tolerierende Form repräsentiert“ (BEREND; KNIPF-KoMLÓSI 2006: 161). Zudem erleichtert die Annahme einer einheitlichen Sprachform das Sprachlernen. Dabei wird aber ignoriert, dass es auch Deutschlerner im Ausland gibt, die bereits durch eine dialektale Form des Deutschen vorgeprägt sind.

Mitte der achtziger Jahre kamen verstärkt Gegenstimmen zu der Sprachbarrierenannahme auf, die den Dialekt als eine gleichberechtigte Varietät in einem sprachlichen Kontinuum sahen, in dem zwischen dem Hochdeutschen und dem Dialekt ein fließender Übergang bestehe und in dem die beiden Varietäten eigene Verwendungsbereiche haben. Der Dialekt sei somit als der Hochsprache ebenbürtig zu betrachten, der den Sprechern, neben einem Plus an Ausdrucksfähigkeit, „Nähe, Vertrautheit, Identifikation, emotionalen Rückhalt und Heimatgefühl“" (SCHIEßL 2009: 36) vermittle, denn die Dialekte spielen für die Sprecher eine bedeutende Rolle als Nähesprachen und als Spiegel der jeweiligen Regionalkultur: „Dialekte sind Zeugen sprach- und kulturhistorischer Traditionen, aus denen die heutige Standardsprache erst in einer über 1000 Jahre alten sprachgeschichtlichen Entwicklung hervorgegangen ist"“ (NEUland; HochHOlzer 2006: 188). Auch Johann Wolfgang von Goethe (1812: 86) sprach schon vom Wert des Dialektes für die Sprecher: „Jede Provinz liebt ihren Dialekt: denn er ist doch eigentlich das Element, in welchem die Seele ihren Atem schöpft“. Die Beherrschung des Dialektes neben der Standardsprache wurde daher nicht mehr als defizitär, sondern vielmehr als bereichernd und vorteilhaft gesehen. Im Zuge der kommunikativen Wende gab es auch Impulse zur Implementierung dialektaler Sprachwirklichkeit in den Deutschunterricht. Die konkrete Umsetzung in Lehrwerken 
und im Sprachunterricht blieb jedoch bis heute eher die Ausnahme (vgl. NEULAND; HOCHHOLZER 2006: 180ff.).

In der Sprachdidaktik wurde ,die Benachteiligung dialektsprechender Kinder [...] als ein Problem eines nicht hinreichend auf solche Probleme vorbereiteten Deutschunterrichts zurückgeführt" (NEulAND; HochHOlzER 2006: 179). Als Konsequenz wurden die dialektbedingten Fehler stärker in den Blickpunkt gerückt. Hierfür wurde das - aus der Fremdsprachendidaktik stammende - Instrument der kontrastiven Grammatik angewandt. Es wurden kontrastive Sprachhefte für acht deutsche Dialektlandschaften herausgegeben, die auf Grundlage der Gegenüberstellung von Hochdeutsch und Dialekt die potenziellen Interferenzen ${ }^{7}$ dialektsprechender Kinder (vor allem in der Orthographie und Grammatik) herausstellen. Diese können nach Theodor Diegritz (1979: 41f.) entweder als Übernahmen sprachlicher Merkmale des Dialekts in das Hochdeutsche auftreten oder aber als Hyperkorrekturen (Übertragung der gelernten Regeln auf Fälle, auf die sie nicht zutreffen).

Die potenziellen Fehlerbereiche könnten nicht nur bei der Gestaltung des Unterrichts helfen, sondern auch dabei, dialektbedingte Fehler als solche zu erkennen und entsprechend zu behandeln. Um das Bewusstsein für die Rechtmäßigkeit des jeweiligen Dialektes zu fördern, wäre es z. B. angebracht, sie weniger streng zu bewerten als andere Fehler. Insgesamt spielen die Einstellungen der Lehrkräfte gegenüber dem Dialektgebrauch eine wesentliche Rolle für das Selbstverständnis dialektsprechender Lerner: „Wichtig ist, daß der Lehrer bei sprachlichen Problemen den Dialekt nicht korrigierend abwertet, sondern vergleichend, kontrastiv hochsprachliche und mundartliche Formen einander gegenüberstellt und so wertfrei die Fehlerursachen aufdeckt" (DIEGRITZ 1979: 44). So ist das von der Mundart durchsetzte gesprochene Hochdeutsche der Lerner als eine Lernersprache zu sehen und nicht zu entwerten. Es sollte auch klar gemacht werden, „dass hochdeutsch sprechen nicht unter den gleichen formalen Anforderungen steht wie hochdeutsch schreiben“ (SIEBER; SITTA 1994: 104). Korrekturen sollten daher nur bei Verständnisschwierigkeiten erfolgen und sich am Sprachstand des einzelnen Lerners orientieren, um sie nicht zu entmutigen, sondern in der von ihnen beherrschten Sprechform zu bestärken und so das Vertrauen in die eigene Sprachfähigkeit stützen. Generell sollten Hochdeutsch und Mundart als gleichwertige

\footnotetext{
${ }^{7}$ Interferenzen sind als unerwünschte Einflüsse der Muttersprache auf die Lernervarietät zu verstehen (vgl. NEUNER 2009: 4).
} 
Ladilova, A. - Wolgadeutsche in Argentinien

Sprechformen gesehen werden und mehr Wert auf den Inhalt gelegt werden (vgl. SIEBER; SitTA 1994: 106). Jürgen STRUGER (2008: 46) plädiert zudem dafür, dass nur geschriebene Texte im Unterricht bewertet werden sollten, denn die Bewertung der mündlichen Sprache sei problematisch, weshalb es lediglich als Hilfsmittel zur Inhaltsvermittlung eingesetzt werden solle.

Es gibt mehrere Möglichkeiten des produktiven Umgangs mit Dialektsprechern im Deutschunterricht. So könnte der Unterricht des Standarddeutschen gestärkt werden, wobei der Dialekt lediglich als Begleiterscheinung des Deutschunterrichts auftreten würde. Eine offensivere Strategie des Umgangs mit Dialekt in der Schule wäre, das Nebeneinander von Standardsprache und Dialekt als sprachliche Realität in den Deutschunterricht zu integrieren. Hierfür müsste den Schülern klar gemacht werden, „dass Standard und Dialekt nicht in einer Bewertungsskala von ,Richtig“ bis ,Falsch“ einzuordnen sind, sondern beide Sprachvarietäten voll funktionsfähige Codes darstellen, die jeweils in verschiedenen Situationen angemessen sind“" (STRUGER 2008: 7). In beiden Fällen müsste der Deutschunterricht in manchen Bereichen kontrastiv angelegt werden. Nach STRUGER (2008: 49f.) treten die dialektalen Unterschiede in der Aussprache wesentlich deutlicher hervor als in der dialektal beeinflussten Schriftsprache des Hochdeutschen, weshalb Hörtraining im Unterricht erforderlich wäre.

Ludwig SCHIEßL (2009: 39f.) plädiert für eine stärkere Implementierung des Dialektes in den Deutschunterricht in Bayern und schlägt vier Möglichkeiten der Anwendung des Dialekts in der Schule vor. Erstens als Kommunikationsmittel, vor allem in privat-persönlichen Kommunikationssituationen. Zweitens als Unterrichtssprache bzw. Metasprache, was vor allem in der Grundschule fruchtbar wäre. Drittens als Unterrichtsgegenstand und viertens als Gegenstand kontrastiver Sprachbetrachtung im Fremdsprachenunterricht. Als Unterrichtsthemen schlägt SCHLIEßL (2009: 45) die Sprachanalyse (Vergleich Hochsprache - Umgangssprache - Dialekt), Textrezeption und -produktion, kulturhistorische Untersuchungen (z. B. als Ortsneckereien) und Projektarbeit (z. B. in Form einer Dialektumfrage) vor. Gleichzeitig müsste auch auf sprachpolitischer Ebene mehr dafür getan werden, um den Dialekt stärker in den Deutschunterricht zu integrieren, denn der gute Wille des Lehrers alleine reicht meist nicht aus.

Peter Sieber und Horst SITTA (1994: 103) setzen sich mit dem Deutschunterricht in der Schweiz auseinander und betonen, dass das Hochdeutsche aufgrund der medialen 
Ladilova, A. - Wolgadeutsche in Argentinien

Präsenz schon beim Schuleintritt keine Fremdsprache für die Schüler sei. Die Autoren sehen es als förderlich für die Lerner, wenn der/die Lehrer/in von Anfang an Hochdeutsch mit ihnen spricht. Bevor die Lerner aber selber Hochdeutsch sprechen, sollte zunächst ihre Verstehenskompetenz gefördert werden, denn das „Verstehen einer Sprache geht dem eigenen Sprechen voraus“" (SIEBER; SITTA 1994: 103). Der Wortschatz sollte in den beiden Varietäten ausgebaut werden, denn „was die Schüler in der einen Sprachform erwerben, wird ihnen zum Besitz auch für die andere“ (SIEBER; SITTA 1994: 103). Hierfür ist die Einbeziehung von Versen, Gedichten, Reimen und Liedern ist sinnvoll, denn sie ermöglicht den spielerischen Erwerb größerer lexikalischer Einheiten. Das Hochdeutsche sollte zudem auf vielfältige Art und Weise (Theater, Rollenspiele, Hörspielrezitation, Kontakte mit fremden Klassen) und in verschiedenen Situationen (in Groß- und Kleingruppen) eingesetzt werden.

Nicht zuletzt soll auf ein neues Konzept aus dem Fremdsprachenlehrbereich hingewiesen werden, das die Verwendung mehrerer Sprach (varietäten) im Unterricht als vorteilhaft für den Lernprozess erachtet. Im Gegensatz präskriptiv-strukturalistisch geprägten Ansätzen aus der Fremdsprachendidaktik, die Transferenzen aus der L1 in die L2 und Code-Switching-Phänomene eher negativ bewerteten, stellt das translanguaging Konzept die sozio-kulturelle Dimension des Lernprozesses in den Vordergrund. Die Verwendung aller Sprachvarietäten, die den Lernern zur Verfügung stehen, soll einen sozialen Raum für die mehrsprachigen Lerner schaffen "by bringing together different dimensions of their personal history, experience and environment, their attitudes, beliefs and performance.” (WEI 2011: 1223). Dies befreit die Lerner vom Zwang, die Sprachen trennen zu müssen oder sich mit soziolinguistischen Sachverhalten (wie Macht, Identität, etc.) auseinander setzen zu müssen (vgl. GARCÍA: 2009), und fördert somit die Kreativität der Lerner.

Selbstverständlich ist der dialektbasierte Hochdeutschunterricht in Deutschland nicht mit Hochdeutschunterricht in Migrationssettings gleichzusetzen, denn die Dachsprache ist unterschiedlich. Im brasilianischen Kontext wurden bereits mehrere Arbeiten zum Hochdeutschunterricht im mehrsprachigen Kontext (PortugiesischHunsrückisch) durchgeführt (vgl. SPINASSÉ 2009; KÄFER 2009), die auch die Sprachbewusstheit ins Zentrum stellen (vgl. KäFER 2013). Inwiefern die für die Dialektsprecher im deutschsprachigen Raum angelegten Vorschläge für den Deutschunterricht auch für den in der vorliegenden Arbeit untersuchten Fall zutreffen, 
wird im vierten und fünften Kapitel besprochen. Zunächst wird aber auf die betroffene Zielgruppe genauer eingegangen.

\section{Die Zielgruppe}

Die Wolgadeutschen in Argentinien haben eine doppelte Migrationsgeschichte erlebt: 1763 sind sie zunächst aus deutschsprachigen Ländern nach Russland und 1874 dann nach Argentinien ausgewandert. In beiden Ländern lebten sie in Sprachinselgemeinschaften, ${ }^{8}$ was den Spracherhalt förderte. Gleichzeitig fanden innerhalb der wolgadeutschen Gemeinschaften Dialektmischungen statt, wobei sich hauptsächlich Merkmale der westmitteldeutschen und vor allem der rheinfränkischen Mundarten durchgesetzt haben (vgl. WIESINGER 1983). Bei der Gegenüberstellung der Arbeiten zum Wolgadeutschen in Russland und Deutschland (vgl. BEREND 1998: 23) und in Argentinien (vgl. SCHMIDT 1997; HIPPERDINGER 2005 dt./1994 span.; JungBLut; PROST RUPPEL 2009; LADILOVA 2013), wird deutlich, dass viele ursprüngliche dialektale Merkmale nicht nur die über 200 Jahre seit der Umsiedlung nach Russland überdauert haben, sondern auch die Migration nach Amerika (vgl. DitTMAR 2009: 60). Die beiden Kontaktsprachen Russisch und Spanisch beeinflussten das Wolgadeutsche hauptsächlich auf lexikalischer Ebene (vgl. HIPPERDINGER 2005 dt./1994 span.: 81; SCHMIDT 1997: 98; LADILOVA 2013: 171-185).

Heute sind zahlreiche kleine wolgadeutsche Siedlungen mit ca. 1000 bis 1500 Einwohnern, auch Kolonien genannt, vor allem im Norden Argentiniens (in den Provinzen Entre Ríos, Buenos Aires und La Pampa) vorzufinden. Trotz der 1950 aus wirtschaftlichen und politischen Gründen eingesetzten Integration in die Mehrheitsgesellschaft, die zum Erwerb des Spanischen führte, wurde in vielen dieser Kolonien das Wolgadeutsche bis heute aufrechterhalten. Auch sonstige kulturelle Eigenarten der Wolgadeutschen werden weiterhin gepflegt. Dies trifft sowohl auf die „plakative Kulturpflege“ in Form von wolgadeutschen Festen, Vereinsaktivitäten sowie Tanz- und Musikgruppen als auch auf den ,wolgadeutschen Charakter“, der sich in einem

\footnotetext{
8 Eine Sprachinsel ist: ,[...] eine durch verhinderte oder verzögerte sprachkulturelle Assimilation entstandene Sprachgemeinschaft, die - als Sprachminderheit von ihrem Hauptgebiet getrennt - durch eine sprachlich / ethnisch differente Mehrheitsgesellschaft umschlossen und / oder überdacht wird, und die sich von der Kontaktgesellschaft durch eine die Sonderheit motivierende soziopsychische Disposition abgrenzt bzw. von ihr ausgegrenzt wird“" (MATTHEIER 1994: 334).
} 
Ladilova, A. - Wolgadeutsche in Argentinien

stärker ausgeprägten Konservatismus, Fleiß, Ordentlichkeit und Familienzusammenhalt, als bei den Argentiniern nicht wolgadeutscher Herkunft äußert.

Insgesamt sind die Wolgadeutschen heutzutage stolz auf ihre ursprüngliche Herkunft, was vor 23 Jahren noch nicht der Fall war. Dies hängt vor allem damit zusammen, dass die Wolgadeutschen im Zuge der Integration in die Mehrheitsgesellschaft stärker diskriminiert wurden, nicht zuletzt aufgrund von schlechten Spanischkenntnissen. Heutzutage sprechen demgegenüber alle Wolgadeutschen einwandfrei Spanisch. Gleichzeitig fing Mitte der neunziger Jahre, im Zusammenhang mit dem hundertjährigen Jubiläum der Wolgadeutschen in Argentinien, ein ethnischer Revitalisierungsprozess an, der sich in einer verstärkten Auseinandersetzung mit der eigenen Herkunft, Kultur und Sprache äußert. Somit kann auch von einer hohen Motivation zum Erwerb des Hochdeutschen ausgegangen werden, denn es ist der Schlüssel zur Urheimat. Zudem sehen die Lerner einen deutlichen Nutzen daran Deutsch zu lernen, denn es bietet ihnen bessere Arbeitsmöglichkeiten (vgl. LADILOVA 2013: 169f.).

Das Hochdeutsche wird von den Mitgliedern der Zielgruppe meist nicht als eine eng mit dem Wolgadeutschen verwandte Varietät gesehen, sondern als eine schwer erlernbare Fremdsprache. ${ }^{9}$ Dies dämpft die Motivation zum Deutscherwerb zusammen mit der Tatsache, dass der Erwerb des Englischen oft als nützlicher wahrgenommen wird. Somit ist es Aufgabe des Deutschunterrichts, die Ähnlichkeiten zwischen dem Hochdeutschen und Wolgadeutschen deutlich zu machen, um somit den Lernern die Angst $\mathrm{zu}$ nehmen und sie $\mathrm{zu}$ motivieren. Das im Zusammenhang mit dem Nationalsozialismus stehende, negative Image Deutschlands und der Deutschen hat bei den Wolgadeutschen dagegen keine Konsequenzen für die Motivation zum Deutscherwerb, denn die Wolgadeutschen identifizieren sich nicht mit den Nationalsozialisten und verspüren daher auch kein Bedürfnis, sich davon zu distanzieren. ${ }^{10}$

Der vorliegende Unterrichtsentwurf richtet sich bewusst an die auf dem Land lebenden Wolgadeutschen, denn diese haben das Wolgadeutsche besser aufrechterhalten als diejenigen, die in den Städten leben. Die Kenntnisse des Wolgadeutschen korrelieren

\footnotetext{
9 Dies hat nicht zuletzt damit zu tun, dass die meisten Wolgadeutschen beim Kontakt mit Hochdeutschsprechern mit ihrer Sprechweise auf Diskriminierung stießen.

${ }^{10}$ Aussage in einem Experteninterview, das im Rahmen der Doktorarbeit der Verfasserin erhoben wurde.
} 
Ladilova, A. - Wolgadeutsche in Argentinien

jedoch auch da negativ mit dem Alter der Sprecher: Während die Älteren es gut sprechen, haben die Jüngeren i. d. R. lediglich eine rezeptive Kompetenz und können einige Versatzstücke auf Wolgadeutsch sprechen. Gleichzeitig haben die jüngeren Wolgadeutschen häufig auch Englischkenntnisse, die die älteren nicht haben. Dies hängt damit zusammen, dass die Jüngeren meist eine höhere Schulbildung haben als die Älteren. Es kann jedoch davon ausgegangen werden, dass alle Lerner lesen und schreiben können.

Da die Durchführung verschiedener, nach Alter und sprachlicher Ausgangssituation getrennter Sprachkurse zurzeit kaum durchsetzbar erscheint, wird von einer heterogenen Lernergruppe ausgegangen. Diese hat zehn bis fünfzehn Teilnehmer, die zwischen 14 und 45 Jahre alt sind ${ }^{11}$ und freiwillig am Unterricht teilnehmen. Die Lerner kennen sich nicht nur gegenseitig, sondern sind teilweise miteinander verwandt, was eine zusätzliche Herausforderung für die Unterrichtsgestaltung darstellt. Das übergeordnete Unterrichtsziel ist, es einen Bogen zwischen dem Wolgadeutschen, als der eigenen Sprache, und dem Hochdeutschen, als der überregionalen Verkehrssprache, zu schlagen. Gleichzeitig soll der Spracherwerb kulturhistorisch eingebettet werden, denn die Lerner sollen sich nicht nur mit der eigenen Geschichte und den eigenen Traditionen auseinandersetzen, sondern auch lernen, wie bzw. wodurch sich die eigene Kultur und Sprache genau von der in Deutschland gelebten und gesprochenen unterscheidet. Das folgende Kapitel geht auf den Unterrichtsentwurf für die ersten 20 Stunden ein, bevor im vorletzten Kapitel der vorliegenden Arbeit eine Doppelstunde detaillierter besprochen wird.

\section{Unterrichtsablauf für die ersten 20 Stunden}

Die Sprachsituation der betroffenen Zielgruppe ähnelt insofern den dialektgeprägten Lernern im deutschsprachigen Raum, als dass sie eine dialektale Varietät des Deutschen in den Deutschunterricht mitbringen. Gleichzeitig unterscheiden sie sich dadurch, dass sie diese unterschiedlich gut beherrschen und zudem das Spanische meist als

\footnotetext{
${ }^{11}$ Diese Einschränkung ergibt sich aus der Überlegung, dass jüngere Teilnehmer kaum das notwendige Sprachbewusstsein dafür haben, aus eigenem Willen einen Deutschkurs zu besuchen, und dass die älteren das Wolgadeutsche gut sprechen und meist kaum Interesse daran haben, die Standardform zu erlernen. Dass dieser Annahme in der Realität widersprochen werden kann, wird jedoch nicht ausgeschlossen.
} 
Muttersprache sprechen. Zudem sind die Lerner unterschiedlich stark mit dem Deutschen in ihrem Alltag konfrontiert. Insofern ist das vorliegende Unterrichtskonzept zwischen Deutsch als Fremdsprache und Deutsch als Muttersprache zu verorten. Die Kenntnisse des Wolgadeutschen sollen dabei nicht nur für den Erwerb des Hochdeutschen fruchtbar gemacht werden, sondern durch die kontrastive Herangehensweise und die Auseinandersetzung mit metasprachlichen Fragestellungen gefördert werden. So soll das Bewusstsein dafür, dass das Wolgadeutsche eine legitime Varietät des Deutschen ist, die ihre Richtigkeit und ihren Wert hat, gestärkt werden. Des Weiteren soll den Lernenden deutlich gemacht werden, dass das Wolgadeutsche mit dem Hochdeutschen durchaus vereinbar ist und für die Lernenden daher keine Fremdsprache im engeren Sinne darstellt. Hierfür sollte im Unterricht eine Auseinandersetzung mit den verschiedenen Varietäten des Deutschen in Deutschland erfolgen.

Gleichzeitig sollen die Lerner für die Unterschiede zwischen dem Wolgadeutschen und dem Hochdeutschen (auf allen Sprachebenen) sensibilisiert werden. Hierzu werden die Besonderheiten des Wolgadeutschen herausgearbeitet (Slavismen, Hispanismen, Dialektmischung, etc.) und dem Hochdeutschen gegenübergestellt. Zudem sollte die Geschichte der Wolgadeutschen, von welcher die meisten Wolgadeutschen in Argentinien nur wenig wissen, sich dafür aber durchaus interessieren, stärker in den Vordergrund gerückt werden.

Es wird teilweise mit dem Lehrwerk Schritte Plus (vgl. NIEBISCH 2009) gearbeitet, wobei viele Themen daraus für die betroffene Zielgruppe weniger relevant sind und daher ausgelassen bzw. durch andere ersetzt werden. Zudem wird mit dem Wörterbuch des Wolgadeutschen (JungBlut; PROST RuPPEL 2009) gearbeitet, das nicht nur die wolgadeutschen Wörter den hochdeutschen und spanischen Äquivalenten gegenüberstellt, sondern im ersten Teil auch sprachstrukturelle (z. B. die kontrastive Betrachtung der wolgadeutschen Phonologie, Grammatik und Lexik) und sprachgeschichtliche Fragestellungen (z. B. bezüglich der Entstehung des Wolgadeutschen und der Entwicklung der Schrift) betrachtet.

Der Unterrichtsentwurf für die ersten 20 Stunden wird in der folgenden Tabelle dargestellt. In der ersten Spalte ist die jeweilige Stundenzahl angegeben, wobei der Unterricht in Doppelstundeneinheiten einmal pro Woche stattfindet. Die zweite Spalte gibt die Thematik der theoretischen Einführung wieder, die am Anfang des Unterrichts etwa 10 bis 20 Minuten in Anspruch nehmen und sich vor allem mit metasprachlichen 
Ladilova, A. - Wolgadeutsche in Argentinien

Aspekten auseinandersetzen soll. Diese erfolgt zunächst auf Spanisch, da dies die Varietät ist, die von allen Lernenden gleich gut beherrscht wird. Im späteren Unterrichtsverlauf kann auch das Deutsche immer stärker in diesen Unterrichtsteil einbezogen werden. In der dritten Spalte wird die praktische Arbeit im Unterricht für die jeweilige Doppelstunde angegeben, die im Allgemeinen insofern lernerorientiert erfolgen soll, als dass bei der Aufgabenstellung auf die jeweilige sprachliche Ausgangssituation der Lerner Rücksicht genommen werden soll, indem mit verschiedenen Texten, Medien und Schwierigkeitsgraden gearbeitet wird. Die vierte Spalte beinhaltet die jeweiligen Hausaufgaben. Diesen wird besonders hoher Stellenwert zugeschrieben, da der Unterricht nur einmal pro Woche stattfindet und die relativ lange Zeit zwischen den Unterrichtseinheiten durch eigenständige Arbeit der Lernenden produktiv genutzt werden soll. Hierzu soll auch von neuen Medien Gebrauch gemacht werden.

Tabelle 1:12 Unterrichtsentwurf für die ersten 20 Stunden $^{13}$

\begin{tabular}{|c|c|c|c|}
\hline Stunde & Theorie & Praxis & Hausaufgaben \\
\hline 1,2 & $\begin{array}{l}\text { Kurze Einfüh- } \\
\text { rung (WD als } \\
\text { Varietät des } \\
\text { Deutschen) }\end{array}$ & $\begin{array}{l}\text { Begrüßen, sich verabschieden, } \\
\text { nach dem Namen fragen (kurze } \\
\text { Dialoge führen); das Alphabet; } \\
\text { Personalpronomen: ich, Du/Sie; } \\
\text { Verb: heißen, sein }\end{array}$ & $\begin{array}{l}\text { Nach Wörtern und } \\
\text { Ausdrücken/Versatz- } \\
\text { stücken im WD suchen }\end{array}$ \\
\hline 3,4 & $\begin{array}{l}\text { Deutsch - eine } \\
\text { germanische } \\
\text { Sprache } \\
\text { (Sprachgeschic } \\
\text { hte) }\end{array}$ & $\begin{array}{l}\text { Wörter und Versatzstücke aus dem } \\
\text { WD ins HD und umgekehrt } \\
\text { übertragen; (gegebenenfalls auch } \\
\text { englische Wörter/Ausdrücke, die } \\
\text { im Deutschen ähnlich sind, } \\
\text { vorstellen); Internationalismen; } \\
\text { nach dem Befinden Fragen und } \\
\text { Befinden ausdrücken; W-Fragen; } \\
\text { Aussagesatz }\end{array}$ & $\begin{array}{l}\text { Nach deutschen } \\
\text { Varietäten recherchieren; } \\
\text { kurze Kennenlerndialoge } \\
\text { schreiben und mit } \\
\text { Freunden und } \\
\text { Familienmitgliedern } \\
\text { durchspielen (dabei auf } \\
\text { Abweichungen in der Le- } \\
\text { xik und Aussprache } \\
\text { achten) }\end{array}$ \\
\hline 5,6 & $\begin{array}{l}\text { Die deutsche } \\
\text { Dialektland- }\end{array}$ & $\begin{array}{l}\text { Dialoge (Hausaufgabe) vorspielen; } \\
\text { Hörverstehen der Wenker Sätze }{ }^{14} \\
\text { in der interaktiven Dialektkarte } \\
\text { (multiple choice); Eigenarten der }\end{array}$ & $\begin{array}{l}\text { Ausspracheübungen des } \\
\text { HD; Rezept eines WD }\end{array}$ \\
\hline
\end{tabular}

${ }^{12}$ Alle Tabellen wurden von der Verfasserin eigenständig erstellt.

${ }^{13}$ WD steht für „Wolgadeutsch/wolgadeutsch“ und HD für „Hochdeutsch/hochdeutsch“ in der Tabelle.

14 Dabei handelt es sich um die von Georg Wenker verwendeten Sätze, die er für die Erstellung der Sprachatlanten der deutschen Lokaldialekte in den Jahren 1876 bis 1887 eingesetzt hat. Dieses Kartenmaterial wurde digitalisiert und in Sprachatlanten der modernen Regionalsprachen des Deutschen integriert (vgl. REDE). 
Ladilova, A. - Wolgadeutsche in Argentinien

\begin{tabular}{|c|c|c|c|}
\hline Stunde & Theorie & Praxis & Hausaufgaben \\
\hline & $\begin{array}{l}\text { schaft (inter- } \\
\text { aktiven Di- } \\
\text { alektkarte) }\end{array}$ & $\begin{array}{l}\text { WD Aussprache (Vokale I); Un- } \\
\text { terrichtssprache }\end{array}$ & $\begin{array}{l}\text { Lieblingsgerichts auf WD } \\
\text { mitbringen }\end{array}$ \\
\hline 7,8 & $\begin{array}{l}\text { Das WD - } \\
\text { lexikalische } \\
\text { Eigenarten }\end{array}$ & $\begin{array}{l}\text { Rezepte (Hausaufgabe) aus dem } \\
\text { WD ins HD übersetzen und } \\
\text { umgekehrt; spanische und } \\
\text { russische Entlehnungen im Dialekt } \\
\text { sowie Entsprechungen im HD; } \\
\text { andere Sprachen; Verb: sprechen, } \\
\text { kochen }\end{array}$ & $\begin{array}{l}\text { Wortschatzübungen im } \\
\text { HD; ältere Freunde und } \\
\text { Familienmitglieder nach } \\
\text { Besonderheiten des WD } \\
\text { Wortschatzes befragen }\end{array}$ \\
\hline 9,10 & $\begin{array}{l}\text { Das WD - } \\
\text { lexikalische } \\
\text { Eigenarten }\end{array}$ & $\begin{array}{l}\text { Archaismen und Eigenbildungen; } \\
\text { etymologische Gründe; Arbeit mit } \\
\text { dem wolgadeutschen Wörterbuch; } \\
\text { Farben }\end{array}$ & $\begin{array}{l}\text { Wortschatzübungen; Ar- } \\
\text { beit mit dem Wörterbuch }\end{array}$ \\
\hline 11,12 & $\begin{array}{l}\text { Das WD - } \\
\text { lexikalische } \\
\text { Eigenarten }\end{array}$ & $\begin{array}{l}\text { Ein WD Lied mit spanischen Un- } \\
\text { tertiteln ins HD übertragen und } \\
\text { umgekehrt; Possessivartikel: } \\
\text { mein/e, dein/e; Zahlen }\end{array}$ & $\begin{array}{l}\text { Bezeichnungen für Fami- } \\
\text { lienmitglieder im WD } \\
\text { suchen }\end{array}$ \\
\hline 13,14 & $\begin{array}{l}\text { Familienbezie- } \\
\text { hungen } \\
\text { Argentinien - } \\
\text { Deutschland }\end{array}$ & $\begin{array}{l}\text { Wortpuzzle: Bezeichnungen für } \\
\text { Familienmitglieder; über die } \\
\text { eigene Familie sprechen; Verb: ha- } \\
\text { ben; Personalpronomen: er/sie, } \\
\text { wir, ihr, sie; Akkusativ }\end{array}$ & $\begin{array}{l}\text { Text über die eigene Fam- } \\
\text { ilie schreiben; über andere } \\
\text { deutsche Minderheiten } \\
\text { auf der Welt recher- } \\
\text { chieren }\end{array}$ \\
\hline 15,16 & $\begin{array}{l}\text { Deutsch auf der } \\
\text { Welt (auch die } \\
\text { deutschen } \\
\text { Minderheiten) }\end{array}$ & $\begin{array}{l}\text { Andere Personen vorstellen (samt } \\
\text { Herkunftsland, Alter und } \\
\text { Sprachkenntnissen); Länder; E- } \\
\text { Mail lesen; Verb: kommen, leben; } \\
\text { Präposition: in, aus }\end{array}$ & $\begin{array}{l}\text { E-Mail schreiben; über } \\
\text { die Geschichte der WD } \\
\text { recherchieren }\end{array}$ \\
\hline 17,18 & $\begin{array}{l}\text { Geschichte der } \\
\text { WD }\end{array}$ & $\begin{array}{l}\text { Bildertext über die Geschichte der } \\
\text { WD in Gruppen lesen (ein aktiver } \\
\text { Dialektsprecher in jeder Gruppe); } \\
\text { zusammengesetztes Perfekt }\end{array}$ & $\begin{array}{l}\text { Einen kurzen Text über } \\
\text { die eigene Familienges- } \\
\text { chichte schreiben }\end{array}$ \\
\hline 19,20 & $\begin{array}{l}\text { Das WD - die } \\
\text { Aussprache }\end{array}$ & $\begin{array}{l}\text { Hörübungen, Eigenarten der WD } \\
\text { Aussprache (Konsonanten I) }\end{array}$ & $\begin{array}{l}\text { Ausspracheübungen im } \\
\text { HD: } \\
\text { Buchstabenergänzungsüb } \\
\text { ung }\end{array}$ \\
\hline
\end{tabular}

Im theoretischen Teil des Unterrichts, der am Anfang der jeweiligen Doppelstunde durch den Lehrenden (der in Interaktion mit den Lernenden steht) erfolgen 
Ladilova, A. - Wolgadeutsche in Argentinien

soll, wird über die Geschichte, die regionale Verteilung und die Eigenarten des Deutschen gesprochen. Dabei wird vor allem auf die Eigenarten sowie auf die Gründe der Abweichung des Wolgadeutschen vom Hochdeutschen eingegangen, wobei hierzu auch die Geschichte der Wolgadeutschen besprochen werden soll. Dies soll das Bewusstsein der Lernenden für die Varianz des Deutschen fördern und somit das sprachliche Selbstbewusstsein bezüglich des Wolgadeutschen stärken.

Im praktischen Teil des Unterrichts werden an erster Stelle die üblichen Fertigkeiten, die am Anfang eines Deutschkurses vorkommen, erlernt. Dazu gehören Begrüßungen, Vorstellungen der eigenen Person und eines anderen Kursteilnehmers, Text- und Hörverstehen von einfachen (Hör-)Texten, (thematische) Wortschatzarbeit, grundlegende Grammatik (Personalpronomen, die wichtigsten Verbformen in Präsens, der zusammengesetzte Perfekt, Präpositionen, etc.). Dabei wird kontrastiv Spanisch Hochdeutsch - Wolgadeutsch (und teilweise auch Englisch) gearbeitet. Gleichzeitig werden die für die Wolgadeutschen in Argentinien relevanten Themen, wie die eigene Familie- oder Gruppengeschichte, die kulturellen Eigenarten der Wolgadeutschen sowie die Gründe der Abweichungen wolgadeutscher Laute, Wortformen und Grammatikkonstruktionen vom Hochdeutschen behandelt. Insgesamt soll die sprachliche Arbeit weitgehend mit der landeskundlichen insofern verknüpft werden, als dass die jeweiligen Abweichungen des Wolgadeutschen von dem Hochdeutschen im jeweiligen soziokulturellen Kontext besprochen werden. Dadurch soll die sprachliche Ausgangsbasis der Lernenden nutzbar gemacht und die Sprachbewusstheit gefördert werden. Zur inneren Differenzierung soll in Gruppen gearbeitet werden und die Aufgabenstellung lernerorientiert erfolgen.

Als Hausaufgabe werden den Lernenden nicht nur Sprachübungen zu den jeweiligen sprachlichen Phänomenen gegeben, sondern es soll ebenfalls der freie Umgang mit dem Deutschen gefördert werden, wobei Recherchearbeit über die jeweils behandelten Themen mit allen den Lernenden zur Verfügung stehenden Mitteln (von Befragung von Familienmitgliedern, Freunden und Nachbarn bis zur Suche in Büchern und im Internet) betrieben wird und somit der Kontakt zu Deutschsprachigen erfolgt.

Im Optimalfall sollten die Lehrer das Wolgadeutsche sprechen, wofür eventuell ein zusätzliches Training erforderlich wäre. Auf jeden Fall sollten sie es verstehen (was durch die relativ geringe Abweichung vom Hochdeutschen nicht zu schwer ist) und die Verwendung des Wolgadeutschen durch die Lernenden im Unterricht unterstützen. Die 
Ladilova, A. - Wolgadeutsche in Argentinien

dialektbedingten Fehler im Hochdeutschen sollten zudem (vor allem am Anfang) weniger streng bewertet werden als andere Fehler. Generell sollte das Wolgadeutsche in der mündlichen Kommunikation neben dem Hochdeutschen verwendet werden, wobei mit der Zeit eine Sensibilisierung für die unterschiedlichen Verwendungsbereiche der beiden Varietäten erfolgen soll.

\section{Materialentwurf für die siebte Doppelstunde}

Die Doppelstunde, auf die in der vorliegenden Arbeit der Fokus gelegt wird, setzt sich thematisch mit der Familie auseinander und hat Wortschatzarbeit im Bereich der Bezeichnungen für Familienmitglieder, die Konjugation des Verbes „,haben“ im Präsens sowie die Personalpronomen, die bis zu diesem Zeitpunkt noch nicht behandelt wurden, zum Gegenstand. Wie auch in anderen Sitzungen soll insofern kontrastiv gearbeitet werden, als dass sowohl die Lexeme als auch die grammatischen Phänomene in den drei Kontaktvarietäten (Hochdeutsch, Wolgadeutsch und Spanisch) erarbeitet werden. Das Material für den praktischen Teil der Stunde wurde dem wolgadeutschen Wörterbuch von Christian Jungblut und Eliana Prost Ruppel (2009) entnommen. Die zeitliche Gliederung der Stunde wird in der folgenden Tabelle veranschaulicht:

Tabelle 2: Zeitliche Gliederung der siebten Unterrichtsstunde.

\begin{tabular}{lll}
\hline Zeit (gesamt) & Unterrichtsabschnitt & Inhalt \\
\hline 5 & Begrüßung & Hallo, Wie geht's Euch heute, etc... \\
\hline $10(15)$ & Theorie: frontal & $\begin{array}{l}\text { Vorstellung der Familienbeziehungen in Deutsch- } \\
\text { land und Argentinien durch die Lehrperson }\end{array}$ \\
\hline $10(25)$ & $\begin{array}{l}\text { Theorie: } \\
\text { Gesamtgruppe }\end{array}$ & $\begin{array}{l}\text { Diskussion mit den Lernenden über die wol- } \\
\text { gadeutschen Familienbeziehungen im Vergleich }\end{array}$ \\
\hline $25(50)$ & Praxis: Arbeit in & Wortpuzzle: Bezeichnungen für \\
& Gruppen & Familienmitglieder \\
\hline $15(65)$ & Praxis: frontal & $\begin{array}{l}\text { Verb: haben; Personalpronomen: er/sie, wir, ihr, } \\
\text { sie }\end{array}$ \\
\hline $15(80)$ & Praxis: Gesamtgruppe & $\begin{array}{l}\text { Mündliche Produktion: Über die eigene Familie } \\
\text { sprechen (Ich habe einen Bruder, Meine Oma } \\
\text { heißt...; etc.) }\end{array}$ \\
& & Auf Wiedersehen, Bis nächste Woche, Tschüss \\
& etc. \\
\hline $10(90)$ & Abschied + Puffer & \\
& &
\end{tabular}

Nach der Begrüßung auf Deutsch (wobei beide Varianten, Hoch- und Wolgadeutsch, erlaubt sind) wird über die Unterschiede in den Familienbeziehungen in 
Ladilova, A. - Wolgadeutsche in Argentinien

Argentinien und in Deutschland von der Lehrperson berichtet. Daraufhin werden diese mit den Lernenden den wolgadeutschen Familienbeziehungen gegenübergestellt. Dies soll das Bewusstsein der Lernenden für die Unterschiede und Ähnlichkeiten der drei in Kontakt stehenden Kulturen (der deutschen, argentinischen und wolgadeutschen) schärfen.

Während der theoretische Teil weitgehend auf Spanisch abgehalten wird (da nicht alle Teilnehmer über ausreichende Kenntnisse des Wolgadeutschen verfügen um es für diesen Unterrichtsabschnitt zu verwenden) und eher landeskundlichen Charakter hat, wird im praktischen Teil so weit wie möglich Deutsch gesprochen. Hierzu wird die in der dritten Doppelstunde (Stunde 5 und 6) behandelte Unterrichtssprache eingesetzt. Die Praxis gliedert sich in drei Abschnitte: Im Ersten wird am in Tabelle 3 dargestellten Wortpuzzle (Bezeichnungen für Familienmitglieder auf Wolgadeutsch, Hochdeutsch und Spanisch) ${ }^{15}$ in Gruppen gearbeitet, wobei in jeder Gruppe mindestens ein/e Sprecher/in des Wolgadeutschen sein sollte, der/die als „Experte/in“ fungiert und den anderen Gruppenmitgliedern bei der Lösung der Aufgabe behilflich sein kann. Ziel ist jedoch, dass er/sie sich zunächst so weit wie möglich zurückhält und nur bei Bedarf eingreift. Somit werden die Bezeichnungen für Familienmitglieder in den drei Kontaktvarietäten spielerisch erarbeitet, was für das Behalten des Gelernten förderlich ist.

Tabelle 3: Wortpuzzle: Familienmitglieder kontrastiv

\begin{tabular}{lll}
\hline Hochdeutsch & Wolgadeutsch & Spanisch \\
\hline Die Eltern & Die Eldere & Los padres \\
\hline Die Enkel(kinder) & Die Engelie /Engelkinrer & los nietos \\
\hline Der Onkel und die Tante & Der Vetter und die Wäes & el tío y la tía \\
\hline Die Schwiegertochter & Die Schnerch & la nuera \\
\hline Die Kinder & Die Kelle oder die Kinrer & los chicos o los niños \\
\hline Die Geschwister & Die Gebrüder und die Gesch- & hermanos y hermanas \\
& wister & \\
\hline Der Cousin und die Cousine & Däs Geschwisterkind & el primo y la prima \\
\hline Die Verwandte & Die Freunde & los parientes \\
\hline Die Großeltern & Die Groußeldere & los abuelos \\
\hline Der Neffe und die Nichte & Däs Bruderschkind und däs & el sobrino y la sobrina \\
& Schwesterkind & \\
\hline Der Schwiegersohn & Der Tochtermann & el yerno \\
\hline Der Freund & Der Sos & el amigo \\
\hline
\end{tabular}
${ }^{15}$ Beim Spanischen wird von der Rio-de-la-Plata Variante ausgegangen, die hauptsächlich in Argentinien
und Uruguay gesprochen wird. 
Ladilova, A. - Wolgadeutsche in Argentinien

Nach dem Puzzle fragt die Lehrperson, welche Unterschiede den Lernenden zwischen dem Hochdeutschen und Wolgadeutschen besonders aufgefallen sind, und geht erklärend darauf ein. So z. B. auf die Tatsache, dass es im Wolgadeutschen eine Bezeichnung für männliche Geschwister gibt, was im Hochdeutschen nicht der Fall ist, oder die Tatsache, dass die Bezeichnungen „Vetter“ und „Base“ auch im Hochdeutschen existieren, dass sie aber mittlerweile nicht mehr Onkel und Tante, sondern Cousin und Cousine bedeuten. Früher wurden sie aber für die Bezeichnung der Geschwister des Vaters (die sich von der Bezeichnung der Geschwister von der Mutter unterschieden) verwendet (vgl. DEACADEMIC). Die Auseinandersetzung mit systemlinguistischen Unterschieden zwischen den drei Kontaktvarietäten fördert den Lerneffekt.

Im zweiten Praxisteil trägt die Lehrperson die Personalpronomen: er/sie, wir, ihr, sie (vgl. Tabelle 4) sowie die Konjugation des Verbes „haben“ (vgl. Tabelle 5) kontrastiv vor.

Tabelle 4: Personalpronomen kontrastiv

\begin{tabular}{lll}
\hline Hochdeutsch & Wolgadeutsch & Rio-de-la-Plata Spanisch \\
\hline Ich & ich & yo \\
\hline Du & Du & vos \\
\hline er, sie, es & der, die, däa & él, ella, - \\
\hline wir & mir & nosotros \\
\hline Ihr & ihr/ehr & - \\
\hline sie, Sie & die, Ihr/Ehr & ellos/as, Ustedes, ustedes \\
\hline
\end{tabular}

Wie schon beim Wortpuzzle fallen die Abweichungen zwischen den beiden Varianten des Deutschen auf. Interessant ist aber auch der systemlinguistische Unterschied zum Rio-de-la-Plata Spanisch, in dem es keine zweite Person Plural im engeren Sinne gibt, denn stattdessen wird die dritte Person Plural verwendet. Dies ist auch bei der Verbkonjugation der Fall:

Tabelle 5: Konjugation des Verbs "haben" kontrastiv

\begin{tabular}{lll}
\hline Hochdeutsch & Wolgadeutsch & Rio-de-la-Plata Spanisch \\
\hline ich habe & ich hun & yo tengo \\
\hline du hast & du host & vos tenés \\
\hline er, sie, es hat & der, die, däs hot & el, ella tiene \\
\hline wie haben & mit häne' & nosotros tenemos \\
\hline ihr habt & ihr, ehr hät & - \\
\hline sie, Sie haben & die, Ihr, Ehr hät & ellos/as, Ustedes, ustedes tie- \\
& & nen \\
\hline
\end{tabular}


Zudem weicht die zweite Person Singular im Rio-de-la-Plata Spanisch vom kastilischen Spanisch ab (und zwar sowohl im Pronomen wie auch im Verb, auch „pronominal-verbaler-voseo “genannt ${ }^{16}$. Die Abweichung im Spanischen sollte an dieser Stelle im Unterricht ebenfalls besprochen werden, denn somit wird das Bewusstsein dafür geschärft, dass sich nicht nur das Deutsche, sondern auch das Spanische regional unterscheidet.

Daraufhin wird das Gelernte in der dritten Praxisphase angewandt, indem in der Gesamtgruppe über die eigene Familie gesprochen wird. Hierfür schreibt die Lehrperson zunächst einige Beispiele an die Tafel wie: „Ich habe einen Bruder/Schwester. Er/sie ist zehn Jahre alt“, oder „Meine Oma heißt Martha und hat graue Haare“ etc. Die Lernenden können daran anknüpfen und einige Sätze mündlich formulieren. Dies ist auch eine Vorbereitung für die Hausaufgabe, denn zu Hause soll ein kurzer Text über die eigene Familie geschrieben werden, in dem ein oder zwei Familienmitglieder vorgestellt werden (z. B. in Bezug auf deren Alter, Sprachkenntnisse und Haarfarbe). Zudem sollen die Lerner als Vorbereitung auf die folgende Sitzung über andere deutsche Minderheiten auf der Welt recherchieren. Hierzu sollten sie von der Lehrperson Hinweise dafür bekommen, dass sie dies tun können, indem sie mit ihren Familienmitgliedern, Freunden und Bekannten darüber sprechen, aber auch indem sie im Internet recherchieren oder sich über die soziale Netzwerke informieren (z. B. indem sie die Facebook Gruppe „Russian Germans International“" auf Deutsch posten).

\section{Zusammenfassung und Ausblick}

Die vorliegende Arbeit setzte sich mit einem Deutschunterrichtskonzept für die Wolgadeutschen in Argentinien auseinander. Hierzu wurde zunächst auf die Problematik des Deutschunterrichts für Dialektsprecher eingegangen, denn die Wolgadeutschen sprechen eine dialektale Varietät des Deutschen - das Wolgadeutsche. Dabei wurde festgestellt, dass, obgleich es einige Konzepte für einen produktiven Umgang mit Dialekt im Deutschunterricht gibt, diese nur selten in die Realität umgesetzt werden. Dies ist auch

\footnotetext{
${ }^{16}$ Zur näheren Information über die Abweichung des Rio-de-la-Plata Spanischen vom Standardkastilischen siehe NOLL (2001).
} 
Ladilova, A. - Wolgadeutsche in Argentinien

bei der Zielgruppe, für die das vorliegende Unterrichtskonzept erstellt wurde, der Fall. Lediglich die älteren Unterrichtskonzepte, die in den wolgadeutschen Siedlungen bis zum Verbot des Deutschunterrichts (in den 1950er Jahren) angewandt wurden, könnten eine stärkere Implementierung des Wolgadeutschen enthalten haben. Um dies zu überprüfen, wäre aber eine mit einer gezielten Feldstudie verbundene Archivarbeit notwendig.

Das Unterrichtskonzept der vorliegenden Arbeit wurde primär kontrastiv gestaltet, und zwar sowohl in Bezug auf die Unterschiede des Wolgadeutschen wie auch des Spanischen vom Hochdeutschen. Beides hilft dabei, den interferenzbedingten Fehlern auf die Spur zu kommen, ihnen durch gezielte Ansprache vorzubeugen und entsprechend $\mathrm{zu}$ bewerten, sowie die Sprachbewusstheit zu fördern. Die Gegenüberstellung des Wolgadeutschen und des Hochdeutschen im Unterricht fördert das Bewusstsein der Sprecher für die eigene Varietät des Deutschen und die kulturhistorische Einbettung macht die Auseinandersetzung mit der eigenen Herkunft möglich. Die positive Einstellung der Lehrperson zum Wolgadeutschen fördert zudem das sprachliche Selbstbewusstsein der Lerner.

Zur Herausstellung konkreter Fehlerbereiche von Wolgadeutschen in Argentinien sollte im besten Fall eine kontrastive Analyse der jeweiligen regionalen Varietät des Wolgadeutschen mit dem Hochdeutschen erfolgen. Da nicht alle möglichen Interferenzen auch auftreten, wäre zudem eine Untersuchung deren tatsächlichen Vorkommens und Häufigkeit vorteilhaft. Hierzu könnte aber auch auf vergleichbare Studien zu anderen deutschen Dialektvarietäten zurückgegriffen werden, denn es gibt eine Reihe von Interferenzbereichen, die sich in mehreren Dialekten vorfinden (vgl. DIEGRITZ 1979: 42f.). Zudem wäre ein Vergleich mit den Erkenntnissen von Studien über Hochdeutschunterricht für Sprecher des Hunsrückischen in Brasilien (KÄFER 2013; 2009; PUPP SPINASSÉ 2009) fruchtbar. Neben solchen Studien zu den Varietäten des Deutschen im Ausland wäre ein stärkerer Einsatz deutscher Organisationen wie der des DAAD oder des Goethe-Instituts für die Durchführung solcher gruppenspezifischeren Deutschkurse wünschenswert.

Neben den im vierten und fünften Kapitel besprochenen Herangehensweisen, die für die ersten 20 Unterrichtsstunden geeignet sind, wäre in einer späteren Phase eine Projektarbeit möglich, in deren Rahmen z. B. eine Dialektumfrage im jeweiligen Dorf durch die Lernenden durchgeführt werden könnte. In diesem Rahmen wären auch Sprachaufnahmen eigener Familienmitglieder (mit dem Handy oder dem MP3-Player) 
Ladilova, A. - Wolgadeutsche in Argentinien

möglich. Anschließend könnte versucht werden, die jeweiligen Dialektmerkmale in die deutsche Dialektlandschaft zu verorten. Zudem könnte die gegenwärtige Lage des Wolgadeutschen erfragt werden, um somit das Bewusstsein dafür zu schärfen, wie stark es im Vergleich zu der Einschätzung der Lerner zu anderen wolgadeutschen Dörfern oder $\mathrm{zu}$ früher zurückgegangen ist. Es könnten auch die Gründe der Verwendung des

Wolgadeutschen in Argentinien erfragt werden, um somit das Bewusstsein für den Wert dieser Varietät für die Sprecher zu stärken.

\section{Literaturverzeichnis}

AMMON, Ulrich. Vorbereitung einer Explizit-Definition von "Dialekt" und benachbarten Begriffen mit Mitteln der formalen Logik. In: MATTHEIER, Klaus J. (ed.): Aspekte der Dialekttheorie. Tübingen: De Gruyter, 1983, p. 27-68.

Association fOR LANGUAGe AwARENESS (ALA), s/d. Disponível em: <http://www.lexically.net/ala/la_defined.htm>. Acesso em: 27 mar. 2012.

BEREND, Nina. Sprachliche Anpassung. Eine soziolinguistisch-dialektologische Untersuchung zum Rußlanddeutschen. Tübingen: Narr, 1998.

BEREND, Nina/KNIPF-KOMLÓsI, Elisabeth. 'Weil die Gegenwartssprache von der Standardsprache abweicht'. Sprachliche Variation als Herausforderung für den Deutschunterricht. In: Neuland, Eva (ed.): Variation im heutigen Deutsch. Perspektiven für den Sprachunterricht. Frankfurt am Main: Lang, 2006, p. 161-174.

BUDDE, Monika. Über Sprache reflektieren. Unterricht in sprachheterogenen Lerngruppen. Fernstudieneinheit 2. Kassel: University Press, 2012.

DEACADEMIC: Disponível em: <http://deacademic.com/dic.nsf/dewiki/1051260>. Acesso em: 11 jan. 2017.

DIEGRITZ, Theodor. Dialekt und Schule. In: NÜNDEL, Ernst Diegritz Theodor et al. (eds.): Sozialintegrative Aspekte des Deutschunterrichts. Donauwörth: Auer, 1979, p. 28-44.

DITTMAR, Martin. Deutsche Sprachinseln in Südamerika: Die wolgadeutsche Mundart in Argentinien. Wissenschaftliche Hausarbeit zur ersten Staatsprüfung für das Lehramt an Gymnasien im Fach Deutsch. Erfurt: Universität, 2009.

FEICK, Diana. Einstellungen zum Deutschen und Möglichkeiten des Spracherhalts in einer wolgadeutschen Sprachinsel in Argentinien. Chancen und Grenzen eines Unterrichts für Deutsch als Fremdsprache/Deutsch als Zweitsprache in diesem Kontext. In: HIPPERDINGER, Yolanda H. (ed.): Estudios sobre contacto inter- e intralingüístico. Bahía Blanca: EDIUNS, 2005, p. 59-120.

GARCÍA, Ofelia. Bilingual Education in the 21st Century: A Global Perspective. Malden, MA: Wiley-Blackwell, 2009.

GoETHE, Johann Wolfgang von. Aus meinem Leben. Dichtung und Wahrheit. Bd. 2., Tübingen: Cotta, 1812.

HIPPERDINGER, Yolanda H. Die Sprache(n) der Wolgadeutschen in Argentinien. Die Kolonisierung des Bezirkes Coronel Suárez. Wien: Praesen, 2005.

Jungblut, Christian/Prost RupPel, Eliana. El Dialecto Alemán del Volga „Das Wolgadeutsch". Alemán del Volga-Alemán Estándar-Español (Wolgadeutsch-Hochdeutsch - Spanisch). El dialecto de los descendientes de Inmigrantes Alemanes del Volga en Argentina registrado en un Diccionario Básico. Bahía Blanca: Selbstverlag, 2009. 
KÄFER, Maria Lidiani. A conscientização linguística como fundamento para uma abordagem plural no ensino de alemão-padrão em contextos de contato português-hunsrückisch. 2013. Dissertação (Mestrado em Letras), Universidade Federal do Rio Grande do Sul, Porto Alegre, 2013. Disponível em: <https://www.lume.ufrgs.br/bitstream/handle/10183/79439/000900613.pdf?sequence=1>. Acesso em: 20.07.2017.

KÄFER, Maria Lidiani. O Ensino de Alemão como LE em contextos bilíngües Português-Hunsrückisch. 2009. Apresentação de Trabalho/Comunicação. Disponível em: <http://hdl.handle.net/10183/42862>. Acesso em: 20.07.2017.

LADILOVA, Anna. Kollektive Identitätskonstruktion in der Migration: Eine Fallstudie zur Sprachkontaktsituation der Wolgadeutschen in Argentinien. Frankfurt: Lang, 2013.

LuCHTENBERG, Sigrid. Language Awareness. In: AHRENHOLZ, Bernt; OOMEN-WelKe, Ingelore (eds.): Deutsch als Zweitsprache. Baltmannsweiler: Schneider-Verl. Hohengehren, 2010, p. 107-117.

MATTHEIER, Klaus J. Theorie der Sprachinsel. Voraussetzungen und Strukturierungen. In: BEREND, Nina/MATTHEIER, Klaus J. (eds.): Sprachinselforschung. Eine Gedenkschrift für Hugo Jedig. Frankfurt am Main: Lang, 1994, p. 333-348.

NEUlAND, Eva. Sprachbewusstsein - eine zentrale Kategorie für den Sprachunterricht. In: Der Deutschunterricht, v. 54, 2002, p. 4-10.

NEUlAND, Eva/HochHOlzER, Rupert. Regionale Sprachvarietäten im muttersprachlichen Deutschunterricht. In: Neuland, Eva (ed.): Variation im heutigen Deutsch. Perspektiven für den Sprachunterricht. Frankfurt am Main: Lang, 2006, p. 175-190.

NEUNER, Gerhard. Mehrsprachigkeitsdidaktik und Tertiärsprachenlernen. Grundlagen - Dimensionen-Merkmale. Zur Konzeption des Lehrwerks ,,deutsch.com “. 2009. Disponível em: <www.hueber.de/mehrsprachigkeitsdidaktik>. Acesso em: 31/03/2017.

NIEBISCH, Daniela. Schritte plus. Deutsch als Fremdsprache. Ismaning: Hueber, 2009.

NoLL, Volker. Das amerikanische Spanisch. Ein regionaler und historischer Überblick. Tübingen: Niemeyer, 2001.

PUPP SPINASSÉ, Karen. O aprendizado do alemão-padrão por alunos bilíngües: pesquisas e ações. In: Contingentia, v. 4, p. 100-109, 2009.

REDE: <https://www.regionalsprache.de>. Acesso em: 18.12.2017.

SCHIEßL, Ludwig. Dialekt und Schule am Beginn des 21. Jahrhunderts. Anspruch und Wirklichkeit unter dem Aspekt neuer wissenschaftlicher Erkenntnisse. In: Ferstl, Christian (ed.): ,Dem Dorfschullehrer sein neues Latein ... '. Beiträge zu Stellenwert und Bedeutung des Dialekts in Erziehung, Unterricht und Wissenschaft. Regensburg: Vulpes, 2009, p. 3248.

SCHMIDT, Arnd. Kollektive Zweisprachigkeit in einsprachiger Umgebung. Eine wolgadeutsche Sprachinsel in Argentinien. Kiel: Westensee, 1997.

SIEBER, Peter/SiTTA, Horst. Mundart und Hochdeutsch im Unterricht. Thesen. In: Sieber, Peter/Sitta, Horst (eds.): Mundart und Hochdeutsch im Unterricht. Orientierungshilfen für Lehrer. Aarau: Sauerländer, 1994, p. 103-107.

STRUGER, Jürgern. ,Das gehört sich nicht'. Umgangssprache, Dialekt und Sprachvarianten im Deutschunterricht - für eine Kultur des sensibilisierten Zuhörens. In: SAXALBER-TETTER, Annemarie (ed.): Individualisierung. Innsbruck/Wien/Bozen: StudienVerlag, 2008, p. 44-51.

WEI, Li. Moment analysis and translanguaging space: Discursive construction of identities by multilingual Chinese youth in Britain. In: Journal of Pragmatics, v. 43, 2011, p. 12221235.

WIESINGER, Peter. Deutsche Dialektgebiete außerhalb des deutschen Sprachgebietes: Mittel-, Südost- und Osteuropa. In: BESCH, Werner et al. (eds.): Dialektologie. Ein Handbuch zur deutschen und allgemeinen Dialektforschung. Berlin: de Gruyter, 1983, p. 900-929. 
Ladilova, A. - Wolgadeutsche in Argentinien

Recebido em 20 de julho de 2017 Aceito em 11 de janeiro de 2018 